\title{
BIFURCATION PROBLEMS FOR THE $p$-LAPLACIAN IN $R^{N}$
}

\author{
PAVEL DRÁBEK AND YIN XI HUANG
}

\author{
ABSTRACT. In this paper we consider the bifurcation problem \\ $-\operatorname{div}\left(|\nabla u|^{p-2} \nabla u\right)=\lambda g(x)|u|^{p-2} u+f(\lambda, x, u)$,
}

in $R^{N}$ with $p>1$. We show that a continuum of positive solutions bifurcates out from the principal eigenvalue $\lambda_{1}$ of the problem

$$
-\operatorname{div}\left(|\nabla u|^{p-2} \nabla u\right)=\lambda g(x)|u|^{p-2} u .
$$

Here both functions $f$ and $g$ may change sign.

\section{INTRODUCTION}

In this paper we consider the following problem in $R^{N}$ :

$$
-\Delta_{p} u=\lambda g(x)|u|^{p-2} u+f(\lambda, x, u),
$$

where $1<p, \lambda \in R, \Delta_{p} u=\operatorname{div}\left(|\nabla u|^{p-2} \nabla u\right)$ is the $p$-Laplacian, both $g$ and $f$ may change sign, and satisfy some conditions to be specified later. We are mainly concerned with the existence of positive solutions to (1.1) for $\lambda$ in certain range. It is known (cf. $[\mathrm{AH}]$ ) that when $g$ satisfies proper conditions, the eigenvalue problem

$$
-\Delta_{p} u=\lambda g(x)|u|^{p-2} u
$$

allows positive eigenvalue $\lambda_{1}$ with positive eigenfunction $u_{1}$. Thus we can study the bifurcation problem when $\lambda$ is near $\lambda_{1}$.

The bifurcation problem of the type (1.1) on bounded domains has received extensive attention recently, and we refer to $[\mathrm{BH}, \mathrm{DM}]$ and $[\mathrm{D} 1, \mathrm{D} 2]$ for details. Topological degree arguments were used there to prove the main results. On the other hand, the study of the existence of global positive solutions of the $p$-Laplacian also sees great increase in number of papers published. We mention [KA1, KA2, KU, LY, SC] and [Y], to name a few. Loosely speaking, most references we mentioned use variational methods, and as such, only the case where (in essence) $g(x)<0, \lambda>0$ and $f(|\lambda|, x,|u|)>0$ was studied thoroughly, and their methods break down when either one of $g$ and $f$ changes sign. For the case $p=2$, [ER] and [RE] studied the bifurcation from the first eigenvalue in $R^{N}$ and obtained the existence of bifurcating branches, where functions $f$ and $g$ were assumed positive, and $f(x, \cdot)$ is sublinear in $[\mathrm{ER}]$ and superlinear in $[\mathrm{RE}]$. We note that topological degree argument and fixed point theory are employed in $[\mathrm{RE}]$ and $[\mathrm{ER}]$ respectively. However, their principal

Received by the editors November 18, 1994 and, in revised form, March 10, 1995.

1991 Mathematics Subject Classification. Primary 35B32, 35J70, 35P30.

Key words and phrases. p-Laplacian, global positive solutions, weighted spaces.

The first author was partially supported by the Grant Agency of the Czech Republic under the Grant No. 201/94/0008. 
operator is defined via a Green's function which is not available to the $p$-Laplacian. In this paper we investigate the situation where both $f$ and $g$ may change sign. Using topological degree arguments, we are able to prove that $\lambda_{1}$ given by [AH] is a bifurcation point of (1.1). In particular, if $g \in L^{N / p} \cap L^{\infty}$ and $g^{ \pm} \not \equiv 0$, we show that there are two bifurcation points. Moreover, under proper conditions, regularity and the decaying of solutions as $|x| \rightarrow \infty$ are obtained, and we also give information on the sign of solutions on the bifurcating branches. We illustrate our results with the following example.

Example. Assume $g(x)=\sin (|x|)(1+|x|)^{-\alpha}$ and $f(x, u)=(1+|x|)^{-a}|u|^{b-2} u$, where $1<p<N, \alpha>p, p<b<N p /(N-p)$ and $a>N-(N-p) b / p$. Then the problem

$$
-\Delta_{p} u=\lambda g(x)|u|^{p-2} u+f(x, u), \text { in } R^{N}
$$

has two positive solution branches containing $\left(\lambda_{1}^{+}, 0\right)$ and $\left(\lambda_{1}^{-}, 0\right)$ in their closures respectively, where $\lambda_{1}^{+}>0$ and $\lambda_{1}^{-}<0$, and $\lambda_{1}^{ \pm}$is the principal eigenvalue of the eigenvalue problem

$$
-\Delta_{p} u=\lambda g|u|^{p-2} u, \text { in } R^{N}, \int( \pm g)|u|^{p}>0 .
$$

Moreover, any solution $u$ of (1.3) belongs to $L^{Q}\left(R^{N}\right)$ for any $N p /(N-p) \leq Q \leq \infty$, and $u$ decays uniformly as $|x| \rightarrow \infty$.

This paper is organized as follows: In section 2 we introduce assumptions and notations which we use extensively in this paper, and prove some technical preliminaries. We note that, in particular, Lemma 2.3 is of its independent interest. In section 3 we verify that the topological degree is well defined for our operators. We then prove our bifurcation theorem in section 4 by showing that the topological degree has a jump when $\lambda$ crosses $\lambda_{1}$. Regularity and decaying property of the solutions, as well as a sign property of the bifurcating branches, are also given in this section.

Acknowledgement. The authors thank the referee for his careful and timely refereeing.

\section{Notations AND PRELIMINARIES}

We study the existence of positive solutions and bifurcation of the problem

$$
-\Delta_{p} u=\lambda g(x)|u|^{p-2} u+f(\lambda, x, u)
$$

in $R^{N}$, with $\lambda \in R$. We first introduce some basic assumptions and notations which we will need in this paper. We assume first that $1<p<N$. Denote $p^{*}=N p /(N-p)$ and $p^{\prime}=p /(p-1)$. Write the weight function $g(x)=g_{1}(x)-g_{2}(x)$ with $g_{1}, g_{2} \geq 0, g_{1} \in L^{\infty}\left(R^{N}\right) \cap L^{N / p}\left(R^{N}\right), g_{2} \in L^{\infty}\left(R^{N}\right)$. We let

$$
\begin{aligned}
\omega(x) & =\frac{1}{(1+|x|)^{p}}, \quad x \in R^{N}, \\
w(x) & =\max \left\{g_{2}(x), \omega(x)\right\}>0, \quad x \in R^{N} .
\end{aligned}
$$


We note here that the function $\omega(x)$ is exactly the weight function in the following Hardy's inequality:

$$
\int_{R^{N}} \frac{|u|^{p}}{(1+|x|)^{p}} \leq\left(\frac{p}{N-p}\right)^{p} \int_{R^{N}}|\nabla u|^{p}
$$

We define the following norm:

$$
\|u\|_{V}=\left(\int|\nabla u|^{p}+\int w(x)|u|^{p}\right)^{1 / p}
$$

where here and henceforth the integrals are taken on the whole $R^{N}$ unless otherwise specified. Let $V$ denote the completion of $C_{0}^{\infty}\left(R^{N}\right)$ with respect to the norm $\|\cdot\|_{V}$, and $V^{*}$ its dual space with the pairing $(\cdot, \cdot)_{V}$. We can see that $V$ is a uniformly convex Banach space. In this paper we denote by $c$ some generic positive constant independent of functions in $V$.

We assume that $f$ satisfies

(f1) $f$ is a Carathéodory function, i.e., $f(\cdot, x, \cdot)$ is continuous for a.e. $x \in R^{N}$ and $f(\lambda, \cdot, u)$ is measurable for all $(\lambda, u) \in R^{2}$;

(f2) $|f(\lambda, x, u)| \leq c(\lambda)\left(\sigma(x)+\rho(x)|u|^{\gamma}\right)$ for a.e. $x \in R^{N}, u \in R$, where $c(\lambda)$ is nonnegative and continuous on $R$, and is bounded on bounded subsets of $R$, $p-1<\gamma<p^{*}-1,0 \leq \rho(x) \in L^{\gamma_{1}}\left(R^{N}\right)$ with $\gamma_{1}=p^{*} /\left(p^{*}-(\gamma+1)\right), 0 \leq$ $\sigma(x) \in L^{N / p}\left(w^{N / p}, R^{N}\right)$, where $L^{N / p}\left(w^{N / p}, R^{N}\right)$ is the weighted $L^{N / p}\left(R^{N}\right)$ space with the weight function $w(x)^{N / p}$, and either

(i) $\sigma(x) \in L^{\left(p^{*}\right)^{\prime}}\left(R^{N}\right),\left(p^{*}\right)^{\prime}=N p /(N p-(N-p))$, or

(ii) $\sigma(x) \in L^{p^{\prime}}\left(w^{1 /(1-p)}, R^{N}\right)$;

(f3) the following limit exists:

$$
\lim _{u \rightarrow 0} \frac{f(\lambda, x, u)}{w(x)|u|^{p-2} u}=0
$$

uniformly for a.e. $x \in R^{N}$ and $\lambda$ in a bounded interval;

We define the operators $J, G, F(\lambda, \cdot): V \rightarrow V^{*}$ as follows: for $u, v \in V$,

$$
\begin{aligned}
(J(u), v)_{V} & =\int|\nabla u|^{p-2} \nabla u \nabla v, \\
(G(u), v)_{V} & =\int g(x)|u|^{p-2} u v, \\
(F(\lambda, u), v)_{V} & =\int f(\lambda, x, u) v .
\end{aligned}
$$

Sometimes we split $G$ as $G=G_{1}-G_{2}$, where

$$
\left(G_{i}(u), v\right)_{V}=\int g_{i}(x)|u|^{p-2} u v, \quad i=1,2 .
$$

We need to establish some properties of these operators for future purposes.

Lemma 2.1. The operators $J, G, F$ are well defined, $G$ and $J$ are $(p-1)$-homogeneous, $J$ is continuous, and $F$ satisfies

$$
\lim _{\|u\|_{V} \rightarrow 0} \frac{\|F(\lambda, u)\|_{V^{*}}}{\|u\|_{V}^{p-1}}=0
$$

uniformly for $\lambda$ in a bounded subset of $R$. 
Proof. (i) Homogeneity of $G, J$ is obvious. Continuity of $J$ follows from the continuity of the Nemytskii operator from $L^{p}\left(R^{N}\right)$ to $L^{p^{\prime}}\left(R^{N}\right)$ (see [FK]).

(ii) Observe that, by Hölder's inequality,

$$
\left|(J(u), v)_{V}\right|=\left.\left|\int\right| \nabla u\right|^{p-2} \nabla u \nabla v \mid \leq\left(\int|\nabla u|^{p}\right)^{1 / p^{\prime}}\left(\int|\nabla v|^{p}\right)^{1 / p}<\infty .
$$

So $J$ is well defined.

(iii) Using Hölder's inequality again, we have

$$
\left|(G(u), v)_{V}\right| \leq\left.\int\left|g^{1 / p^{\prime}}\right| u\right|^{p-2} u \cdot g^{1 / p} v \mid \leq\left(\int|g| \cdot|u|^{p}\right)^{1 / p^{\prime}} \cdot\left(\int|g| \cdot|v|^{p}\right)^{1 / p} .
$$

Note that for $u \in V, \int g_{2}|u|^{p}<\infty$ by the definition of $V$, and by Hölder's inequality, assumption on $g_{1}$ and the Sobolev embedding theorem (see, e.g. [AD]), we have

$$
\int g_{1}|u|^{p} \leq\left(\int g_{1}^{N / p}\right)^{p / N} \cdot\left(\int|u|^{p^{*}}\right)^{p / p^{*}}<\infty .
$$

Hence $G$ is well defined.

(iv) For $F$, we have

$$
\left|(F(u), v)_{V}\right|=\left|\int f(\lambda, x, u) v\right| \leq c(\lambda)\left(\int \sigma|v|+\int \rho|u|^{\gamma}|v|\right) .
$$

Now, by (f2), either

$$
\int \sigma|v| \leq\left(\int \sigma^{\left(p^{*}\right)^{\prime}}\right)^{1 /\left(p^{*}\right)^{\prime}}\left(\int|v|^{p^{*}}\right)^{1 / p^{*}}<\infty,
$$

or

$$
\int \sigma|v| \leq\left(\int w^{1 /(1-p)} \sigma^{p^{\prime}}\right)^{1 / p^{\prime}}\left(\int w|v|^{p}\right)^{1 / p}<\infty
$$

and

$$
\begin{aligned}
\int \rho|u|^{\gamma}|v| & \leq\left(\int|u|^{p^{*}}\right)^{\gamma / p^{*}} \cdot\left(\int \rho^{p^{*} /\left(p^{*}-\gamma\right)}|v|^{p^{*} /\left(p^{*}-\gamma\right)}\right)^{\left(p^{*}-\gamma\right) / p^{*}} \\
& \leq c_{1}\left(\int|u|^{p^{*}}\right)^{\gamma / p^{*}} \cdot\left(\int|v|^{p^{*}}\right)^{1 / p^{*}} \cdot\left(\int \rho^{\gamma_{1}}\right)^{1 / \gamma_{1}} \\
& \leq c_{2}\|v\|_{V} \cdot\|u\|_{V}^{\gamma}<\infty ;
\end{aligned}
$$

thus $F$ is well defined.

(v) By definition,

$$
\begin{aligned}
\lim _{\|u\|_{V} \rightarrow 0} \frac{\|F(\lambda, u)\|_{V^{*}}}{\|u\|_{V}^{p-1}} & =\lim _{\|u\|_{V} \rightarrow 0} \sup _{\|v\|_{V} \leq 1} \frac{1}{\|u\|_{V}^{p-1}}\left|\int f(\lambda, x, u) v\right| \\
& \leq \lim _{\|u\|_{V} \rightarrow 0} \sup _{\|v\|_{V} \leq 1} \int \frac{|f(\lambda, x, u)|}{w|u|^{p-1}}|\tilde{u}|^{p-1}|v| w,
\end{aligned}
$$

where $\tilde{u}=u /\|u\|_{V}$. We now estimate the integral.

We first define, for $\delta>0$,

$$
\Omega_{\delta}(u)=\left\{x \in R^{N}: w(x)|u(x)|^{p-1} \geq \delta\right\} .
$$


We claim that, as $\|u\|_{V} \rightarrow 0$, meas $\Omega_{\delta}(u) \rightarrow 0$. Assume, on the contrary, meas $\Omega_{\delta}(u)$ $\geq c_{3}>0$. Let $\Omega_{K}=\Omega_{\delta}(u) \cap B_{K}(0)$ for $K>0$. Then meas $\Omega_{K} \geq c_{3} / 2$ taking $K>0$ large enough. Hence we have

$$
\begin{aligned}
0 & <\delta \text { meas } \Omega_{K} \leq \int_{\Omega_{K}} w(x)|u(x)|^{p-1} \leq\left(\int_{\Omega_{K}} w(x)|u(x)|^{p}\right)^{1 / p^{\prime}} \cdot\left(\int_{\Omega_{K}} w(x)\right)^{1 / p} \\
& \leq\left(\int_{\Omega_{K}} w(x)\right)^{1 / p} \cdot\|u\|_{V}^{p-1} \leq c_{4}\left(\text { meas } \Omega_{K}\right)^{1 / p} \cdot\|u\|_{V}^{p-1} .
\end{aligned}
$$

That is,

$$
0<\delta\left[\frac{c_{3}}{2}\right]^{1 / p^{\prime}}<\delta\left(\text { meas } \Omega_{K}\right)^{1 / p^{\prime}} \leq c_{4}\|u\|_{V}^{p-1}
$$

a contradiction. Now for any given $\varepsilon>0$, by (f3), there exists $\delta>0$ so that

$$
\frac{|f(\lambda, x, u)|}{w(x)|u|^{p-1}} \leq \varepsilon
$$

uniformly for $w(x)|u|^{p-1}<\delta$. We split the integral in (2.4) into integrals on $R^{N} \backslash \Omega_{\delta}(u)$ and $\Omega_{\delta}(u)$ respectively. For the first integral, we have

$$
\int_{R^{N} \backslash \Omega_{\delta}(u)} \frac{|f(\lambda, x, u)|}{w(x)|u|^{p-1}}|\tilde{u}|^{p-1}|v| w \leq \varepsilon \int_{R^{N} \backslash \Omega_{\delta}(u)}|\tilde{u}|^{p-1}|v| w \leq c_{5} \varepsilon,
$$

where we have used the following inequality:

$$
\int|\tilde{u}|^{p-1}|v| w \leq\left(\int w|\tilde{u}|^{p}\right)^{1 / p^{\prime}} \cdot\left(\int w|v|^{p}\right)^{1 / p}<\infty .
$$

By (f2), we have, for the second integral,

$$
\begin{aligned}
\int_{\Omega_{\delta}(u)} \frac{|f(\lambda, x, u)|}{w(x)|u|^{p-1}}|\tilde{u}|^{p-1}|v| w & \leq \int_{\Omega_{\delta}(u)} \frac{c(\lambda) \sigma(x)}{w(x)|u|^{p-1}}|\tilde{u}|^{p-1}|v| w \\
& +\frac{c(\lambda)}{\|u\|_{V}^{p-1}} \int_{\Omega_{\delta}(u)} \rho(x)|u|^{\gamma}|v|:=c(\lambda)\left(I_{1}+I_{2}\right) .
\end{aligned}
$$

Observe that

$$
\begin{aligned}
I_{1} & \leq \frac{1}{\delta} \int_{\Omega_{\delta}(u)} \sigma(x) w(x)|\tilde{u}|^{p-1}|v| \\
& \leq \frac{1}{\delta}\left(\int_{\Omega_{\delta}(u)}|\tilde{u}|^{p *}\right)^{(p-1) / p *} \cdot\left(\int_{\Omega_{\delta}(u)}(\sigma(x) w(x) \cdot|v|)^{p * /(p *-(p-1))}\right)^{(p *-(p-1)) / p *} \\
& \leq \frac{1}{\delta}\left(\int_{\Omega_{\delta}(u)}|\tilde{u}|^{p *}\right)^{(p-1) / p *}\left(\int_{\Omega_{\delta}(u)}(\sigma(x) w(x))^{p * /(p *-p)}\right)^{(p *-p) / p *}\left(\int_{\Omega_{\delta}(u)}|v|^{p *}\right)^{1 / p *} \\
& \leq c_{6}\left(\int_{\Omega_{\delta}(u)}(\sigma(x) w(x))^{N / p}\right)^{p / N}\|\tilde{u}\|_{V}^{p-1}\|v\|_{V} \rightarrow 0,
\end{aligned}
$$

since meas $\Omega_{\delta}(u) \rightarrow 0$ and $\sigma \in L^{N / p}\left(w^{N / p}, R^{N}\right)$. The estimate (2.3) implies

$$
I_{2} \leq c_{2}\|u\|_{V}^{\gamma-p+1}\|v\|_{V} \rightarrow 0
$$

We thus conclude that (2.2) holds. This completes the proof.

Lemma 2.2. (i) The operator $G_{2}$ is continuous. (ii) The operators $G_{1}$ and $F$ are compact. 
Proof. (i) Continuity of $G_{2}$ follows from the continuity of the Nemytskii operator from weighted space $L^{p}\left(w, R^{N}\right)$ to $L^{p^{\prime}}\left(w, R^{N}\right)$.

(ii1) Compactness of $G_{1}$. We first claim that, for any $\varepsilon>0$ and $\varphi \in V$, there exists $K>0$, such that

$$
\sup _{\|v\|_{V} \leq 1} \int_{|x|>K} g_{1}|\varphi|^{p-1}|v| \leq \varepsilon\|\varphi\|_{V}^{p-1}
$$

Indeed, by Hölder's inequality,

$$
\begin{aligned}
\sup _{\|v\|_{V} \leq 1} & \int_{|x|>K} g_{1}|\varphi|^{p-1}|v| \leq \sup _{\|v\|_{V} \leq 1}\left(\int_{|x|>K} g_{1}|\varphi|^{p}\right)^{1 / p^{\prime}} \cdot\left(\int_{|x|>K} g_{1}|v|^{p}\right)^{1 / p} \\
\leq & \sup _{\|v\|_{V} \leq 1}\left(\int_{|x|>K} g_{1}^{N / p}\right)^{(p-1) / N}\left(\int_{|x|>K}|\varphi|^{p^{*}}\right)^{(p-1) / p^{*}} \\
& \cdot\left(\int_{|x|>K} g_{1}^{N / p}\right)^{1 / N}\left(\int_{|x|>K}|v|^{p^{*}}\right)^{1 / p^{*}} \\
\leq & c_{7} \sup _{\|v\|_{V} \leq 1}\left(\int_{|x|>K} g_{1}^{N / p}\right)^{p / N}\left(\int_{|x|>K}|\varphi|^{p^{*}}\right)^{(p-1) / p^{*}}\|v\|_{V} \\
\leq & \varepsilon\|\varphi\|_{V}^{p-1},
\end{aligned}
$$

since $g_{1} \in L^{N / p}$.

Now, suppose $u_{n} \rightarrow u_{0}$ weakly in $V$. We estimate

$$
\begin{aligned}
& \left\|G_{1}\left(u_{n}\right)-G_{1}\left(u_{0}\right)\right\|_{V^{*}}=\sup _{\|v\|_{V} \leq 1}\left|\left(G_{1}\left(u_{n}\right)-G_{1}\left(u_{0}\right), v\right)_{V}\right| \\
& \quad=\sup _{\|v\|_{V} \leq 1}\left|\int g_{1}(x)\left(\left|u_{n}\right|^{p-2} u_{n}-\left|u_{0}\right|^{p-2} u_{0}\right) v\right| \\
& \leq \sup _{\|v\|_{V} \leq 1}\left|\int_{|x| \leq K} g_{1}(x)\left(\left|u_{n}\right|^{p-2} u_{n}-\left|u_{0}\right|^{p-2} u_{0}\right) v\right| \\
& \quad+\sup _{\|v\|_{V} \leq 1}\left|\int_{|x|>K} g_{1}(x)\left(\left|u_{n}\right|^{p-2} u_{n}-\left|u_{0}\right|^{p-2} u_{0}\right) v\right| .
\end{aligned}
$$

Observe that for any $\varepsilon>0$, we can choose a $K>0$ so that the integral over $(|x|>K)$ is smaller than $\varepsilon / 2$ for all $n$, while for this fixed $K$, by strong convergence of $u_{n}$ to $u_{0}$ in $L^{p^{*}}$ on any bounded region, the integral over $(|x| \leq K)$ is smaller than $\varepsilon / 2$ for $n$ large enough. We thus have proved that $G_{1}\left(u_{n}\right) \rightarrow G_{1}\left(u_{0}\right)$ strongly in $V^{*}$, i.e. $G_{1}$ is compact.

(ii2) Compactness of $F$. Let again $u_{n} \rightarrow u_{0}$ weakly in $V$. We have

$$
\begin{aligned}
& \sup _{\|v\|_{V} \leq 1}\left|\left(F\left(\lambda, u_{n}\right)-F\left(\lambda, u_{0}\right), v\right)_{V}\right| \\
& \quad=\sup _{\|v\|_{V} \leq 1}\left|\int\left(f\left(\lambda, x, u_{n}\right)-f\left(\lambda, x, u_{0}\right)\right) v\right| \\
& \leq \sup _{\|v\|_{V} \leq 1}\left|\int_{|x| \leq K}\left(f\left(\lambda, x, u_{n}\right)-f\left(\lambda, x, u_{0}\right)\right) v\right| \\
& +\sup _{\|v\|_{V} \leq 1}\left|\int_{|x|>K}\left(f\left(\lambda, x, u_{n}\right)-f\left(\lambda, x, u_{0}\right)\right) v\right| .
\end{aligned}
$$

It is easy to see that the integral over $(|x| \leq K)$ tends to zero as $n \rightarrow \infty$ by the continuity of the Nemytskii operator $F$ from $L^{p}(|x| \leq K)$ into $L^{p^{\prime}}(|x| \leq K)$. We 
estimate the integral over $(|x|>K)$, as for the case of $G_{1}$,

$$
\sup _{\|v\|_{V} \leq 1}\left|\int_{|x|>K} f(\lambda, x, \varphi) v\right| \leq \sup _{\|v\|_{V} \leq 1} \int_{|x|>K} c(\lambda) \sigma|v|+\sup _{\|v\|_{V} \leq 1} \int_{|x|>K} c(\lambda) \rho|\varphi|^{\gamma} v .
$$

The first term is estimated by

$$
c_{8}\left(\int_{|x|>K} \sigma^{\left(p^{*}\right)^{\prime}}\right)^{1 /\left(p^{*}\right)^{\prime}}, \quad \text { or by } \quad c_{8}\left(\int_{|x|>K} w^{1 /(1-p)} \sigma^{p^{\prime}}\right)^{1 / p^{\prime}},
$$

respectively, and the second term is estimated by

$$
c_{9}\left(\int|\varphi|^{p^{*}}\right)^{\gamma / p^{*}} \cdot\left(\int|v|^{p^{*}}\right)^{1 / p^{*}} \cdot\left(\int_{|x|>K} \rho^{\gamma_{1}}\right)^{1 / \gamma_{1}},
$$

as in the proof of (iv) of Lemma 2.1. We derive then an inequality like (2.5) which in turn implies that the integral over $(|x|>K)$ in (2.6) tends to zero. Thus $F$ is compact. The proof is complete.

Now we give

Definition 2.1. We say that $\lambda \in R$ and $u \in V$ solve the problem (2.1) if

$$
J(u)-\lambda G(u)-F(\lambda, u)=0 \quad \text { in } V^{*} .
$$

We recall that, for $g$ satisfying the aforementioned conditions, the eigenvalue problem

$$
-\Delta_{p} u=\lambda g(x)|u|^{p-2} u
$$

has a pair of principal eigenvalue and eigenfunction $\left(\lambda_{1}^{+}, u_{1}^{+}\right)$with $\lambda_{1}^{+}>0$ and $0<u_{1}^{+} \in V$. Moreover, such $\lambda_{1}^{+}$is simple, unique. If $g_{2} \not \equiv 0$ and $g_{i} \in L^{\infty}\left(R^{N}\right) \cap$ $L^{N / p}\left(R^{N}\right), i=1,2$, then by symmetry there is also principal eigenpair $\left(\lambda_{1}^{-}, u_{1}^{-}\right)$ with $\lambda_{1}^{-}<0$ and $0<u_{1}^{-} \in V$ with analogous properties. For details, we refer to [AH] for $p \neq 2$ and [ER] for $p=2$. Moreover, we will use the following important properties of $\left(\lambda_{1}^{ \pm}, u_{1}^{ \pm}\right)$. For the sake of brevity we will formulate everything in terms of $\lambda_{1}^{+}$and $u_{1}^{+}$respectively. The situation for $\lambda_{1}^{-}$(if it exists) is similar by symmetry.

Lemma 2.3. (i) Every eigenfunction corresponding to the eigenvalue $0<\lambda_{0} \neq \lambda_{1}^{+}$ changes sign in $R^{N}$. (ii) The principal eigenvalue $\lambda_{1}^{+}>0$ is isolated.

Proof. (i). Assume at first that $u_{0}>0$ is the eigenfunction associated with $\lambda_{0}$ of (2.8) with $0<\lambda_{0} \neq \lambda_{1}^{+}$. Then $\lambda_{0}>\lambda_{1}^{+}$by the variational characterization of the principal eigenvalue $\lambda_{1}^{+}>0$. Then by using (2.8) we have, similarly as in [A] and [LQ],

$$
0 \leq\left(\lambda_{0}-\lambda_{1}^{+}\right) \int g(x)\left(u_{0}^{p}-\left(u_{1}^{+}\right)^{p}\right) .
$$

Renormalizing $u_{0}$ so that the last integral is negative we derive a contradiction. Hence $u_{0}$ must change sign.

(ii). Let $\left(\lambda_{0}, u_{0}\right)$ be as in (i) and $\lambda_{0}$ belong to some fixed neighbourhood of $\lambda_{1}$. Denote $\Omega_{0}^{-}=\left\{x \in R^{N}: u_{0}(x)<0\right\}$. Then it follows from (2.8) (with $\lambda=\lambda_{0}$ and $\left.u=u_{0}\right)$ that

$$
\int\left|\nabla u_{0}^{-}\right|^{p}+\lambda_{0} \int g_{2}(x)\left|u_{0}^{-}\right|^{p}=\lambda_{0} \int g_{1}(x)\left|u_{0}^{-}\right|^{p}
$$


By Hardy's and Hölder's inequalities, the definition of the norm in $V$, and the assumption on $g_{1}$, we derive from this that

$$
c_{10}\left\|u_{0}^{-}\right\|_{V}^{p} \leq\left(\int_{\Omega_{0}^{-}} g_{1}^{N / p}(x)\right)^{p / N}\left\|u_{0}^{-}\right\|_{L^{p^{*}}\left(R^{N}\right)}^{p} .
$$

Consequently we obtain, by the Sobolev embedding,

$$
\left(\int_{\Omega_{0}^{-}} g_{1}^{N / p}(x)\right)^{p / N} \geq c_{11}>0 .
$$

(Independently of $\lambda_{0}$ and $u_{0}$ ). In particular it follows from (2.9) that taking $K_{0}>0$ large enough we have

$$
\text { meas }\left(\Omega_{0}^{-} \cap B_{K}(0)\right) \geq c_{12}
$$

for any $K \geq K_{0}$, where $c_{12}>0$ depends neither on $\lambda_{0}$ nor on $u_{0}$.

Suppose, now, there exists a sequence of eigenpairs $\left(\lambda_{n}, u_{n}\right)$ of $(2.8)$ with $\lambda_{n} \rightarrow$ $\lambda_{1}^{+}$. Then $\lambda_{n}>\lambda_{1}^{+}$and without loss of generality we may assume that $\left\|u_{n}\right\|_{V}=1$ and $u_{n} \rightarrow \tilde{u}$ weakly in $V$ for some $\tilde{u} \in V$. By this weak convergence and simplicity of $\lambda_{1}^{+}$we get from (2.8) that either $\tilde{u}=u_{1}^{+}$or $\tilde{u}=-u_{1}^{+}$. Assume further that $u_{n} \rightarrow u_{1}^{+}>0$ weakly in $V$. It follows from (2.8) that

$$
\begin{aligned}
& \int\left(\left|\nabla u_{n}\right|^{p-2} \nabla u_{n}-\left|\nabla u_{m}\right|^{p-2} \nabla u_{m}\right) \nabla\left(u_{n}-u_{m}\right) \\
& =\int \lambda_{n} g(x)\left(\left|u_{n}\right|^{p-2} u_{n}-\left|u_{m}\right|^{p-2} u_{m}\right)\left(u_{n}-u_{m}\right) \\
& \quad+\left(\lambda_{n}-\lambda_{m}\right) \int g(x)\left(\left|u_{n}\right|^{p-2} u_{n}-\left|u_{m}\right|^{p-2} u_{m}\right)\left(u_{n}-u_{m}\right) .
\end{aligned}
$$

We then deduce

$$
\begin{aligned}
& \int\left(\left|\nabla u_{n}\right|^{p-2} \nabla u_{n}-\left|\nabla u_{m}\right|^{p-2} \nabla u_{m}\right) \nabla\left(u_{n}-u_{m}\right) \\
& \leq \int_{|x| \leq K} \lambda_{n} g_{1}(x)\left(\left|u_{n}\right|^{p-2} u_{n}-\left|u_{m}\right|^{p-2} u_{m}\right)\left(u_{n}-u_{m}\right) \\
& \quad+\int_{|x|>K} \lambda_{n} g_{1}(x)\left(\left|u_{n}\right|^{p-2} u_{n}-\left|u_{m}\right|^{p-2} u_{m}\right)\left(u_{n}-u_{m}\right) \\
& \quad+c_{13}\left|\lambda_{n}-\lambda_{m}\right|\left(\left\|u_{n}\right\|_{V}^{p}+\left\|u_{m}\right\|_{V}^{p}\right) \rightarrow 0 .
\end{aligned}
$$

Observe that, for any $w, v \in L^{p}\left(R^{N}\right)$,

$$
\begin{aligned}
\int\left(|w|^{p-2} w-|v|^{p-2} v\right) \cdot(w-v) \\
\quad=\int\left(|w|^{p}+|v|^{p}-|w|^{p-2} w v-|v|^{p-2} v w\right) \\
\quad \geq \int\left(|w|^{p}+|v|^{p}\right)-\left(\int|w|^{p}\right)^{1 / p^{\prime}} \cdot\left(\int|v|^{p}\right)^{1 / p}-\left(\int|w|^{p}\right)^{1 / p} \cdot\left(\int|v|^{p}\right)^{1 / p^{\prime}} \\
\quad=\left[\left(\int|w|^{p}\right)^{(p-1) / p}-\left(\int|v|^{p}\right)^{(p-1) / p}\right] \cdot\left[\left(\int|w|^{p}\right)^{1 / p}-\left(\int|v|^{p}\right)^{1 / p}\right] \geq 0 .
\end{aligned}
$$

We then derive from (2.11) and (2.12) that $\int\left|\nabla u_{n}\right|^{p} \rightarrow \int\left|\nabla u_{1}^{+}\right|^{p}$. Hence $\nabla u_{n} \rightarrow$ $\nabla u_{1}^{+}$strongly in $L^{p}\left(R^{N}\right)$. By Hardy's inequality and (2.8) we have then $u_{n} \rightarrow u_{1}^{+}$ 
strongly in $L^{p}\left(w, R^{N}\right)$. Thus we conclude that $u_{n} \rightarrow u_{1}^{+}$strongly in $V$. Fix $K \geq K_{0}$. Then we also have $u_{n} \rightarrow u_{1}^{+}$strongly in $W^{1, p}\left(B_{K}(0)\right)$. By Egorov's Theorem (see, e.g. [HS]), $u_{n}$ converges uniformly to $u_{1}^{+}$on $B_{K}(0)$ with the exception of the set with arbitrarily small measure. But this contradicts (2.10), replacing subscript 0 there by $n$ (cf. [A]). Thus we have proved that $\lambda_{1}^{+}$is isolated. This proves the lemma.

Remark 2.1. For the case $p \geq N$, by a result in [AH], in order that $\lambda_{1}^{+}>0$ exists, we have to assume $g_{1}(x) \not \equiv 0, g_{1} \in L^{N_{0} / p}\left(R^{N}\right)$ for some $N_{0}>p$, and moreover, $g_{2}(x) \geq \varepsilon>0$ in $R^{N}$, which implies in particular that $\lambda_{1}^{-}$does not exist. Observe that in this case we can assume $g_{2}(x) \geq \omega(x)$ so the norm in $V$ is

$$
\|u\|_{V}=\left(\int|\nabla u|^{p}+\int g_{2}(x)|u|^{p}\right)^{1 / p} .
$$

In the assumptions, we replace $p^{*}$ by $\tilde{p}=N_{0} p /\left(N_{0}-p\right)$, assume $\sigma(x) \in L^{N_{0} / p}\left(R^{N}\right)$, and either (i) $\sigma(x) \in L^{(\tilde{p})^{\prime}}\left(R^{N}\right)$, or (ii) $\sigma(x) \in L^{p^{\prime}}\left(R^{N}\right)$, where $(\tilde{p})^{\prime}=\tilde{p} /(\tilde{p}-1)$. Observing that

$$
\left(\int|u|^{\tilde{p}}\right)^{1 / \tilde{p}} \leq c_{14}\|u\|_{V}
$$

by Sobolev's embedding theorem, we see that the proofs in this section can be carried over and the conclusions remain valid for the case $p \geq N$.

\section{Topological DEGREe}

In this section we will define the topological degrees for the operators given by the left-hand side of (2.7).

We first recall some known results about the degree theory for operators from a Banach space $X$ to $X^{*}$. Let $X$ be a real reflexive Banach space and $X^{*}$ its dual, and $A: X \rightarrow X^{*}$ be a demicontinuous operator (cf. [FK]). We assume $A$ satisfies the condition $\alpha(X)$, i.e.

for any sequence $u_{n} \in X$ satisfying $u_{n} \rightarrow u_{0}$ weakly in $X$ and

$$
\lim \sup \left(A\left(u_{n}\right), u_{n}-u_{0}\right)_{X} \leq 0
$$

$$
u_{n} \rightarrow u_{0} \text { strongly in } X \text {. }
$$

Then it is possible to define the degree $\operatorname{Deg}[A ; D, 0]$, where $D \subset X$ is a bounded open set such that $A(u) \neq 0$ for any $u \in \partial D$. Its properties are proved in [SK] or in [BP] (where $\alpha(X)$ is called $(S)_{+}$). Note that the properties are analogous to the ones of the Leray-Schauder degree and we recall some of them which will be used in our proof.

A point $u_{0} \in X$ will be called a critical point of $A$ if $A\left(u_{0}\right)=0$. We say that $u_{0}$ is an isolated critical point of $A$ if there exists $\varepsilon>0$ such that for any $u \in B_{\varepsilon}\left(u_{0}\right)$, $A(u) \neq 0$ if $u \neq u_{0}$. Then the limit

$$
\text { Ind }\left(A, u_{0}\right)=\lim _{\varepsilon \rightarrow 0} \operatorname{Deg}\left[A ; B_{\varepsilon}\left(u_{0}\right), 0\right]
$$

exists and is called the index of the isolated critical point $u_{0}$ of $A$.

Assume furthermore that $A$ is a potential operator, i.e., for some continuously differentiable functional $\Phi: X \rightarrow R, \Phi^{\prime}(u)=A(u), u \in X$. Then we have the following two lemmas. (For their proofs and other related properties of the degree "Deg," we refer to the book [SK].) 
Lemma 3.1. Let $u_{0}$ be a local mimimum of $\Phi$ and an isolated critical point of $A$. Then

$$
\operatorname{Ind}\left(A, u_{0}\right)=1 .
$$

Lemma 3.2. Assume that $(A(u), u)_{X}>0$ for all $u \in X,\|u\|_{X}=r$. Then

$$
\operatorname{Deg}\left[A ; B_{r}(0), 0\right]=1 .
$$

Remark 3.1. Note that these assertions are natural extensions of analogous properties of the Brouwer degree.

Remark 3.2. Note that every continuous map $A: X \rightarrow X^{*}$ is also demicontinuous. Note also, that if $A$ satisfies the condition $\alpha(X)$ then $A+K$ also satisfies the condition $\alpha(X)$ for any compact operator $K: X \rightarrow X^{*}$.

Now we take $X=V$ and $A_{\lambda}=J-\lambda G-F(\lambda, \cdot)$. We verify in this section that the degree "Deg" is defined for the operator $A_{\lambda}$. By Lemmas 2.1, 2.2 and Remark 3.2 , it's sufficient to prove

Lemma 3.3. The operator $J+\lambda G_{2}: V \rightarrow V^{*}$ satisfies $\alpha(V)$ for $\lambda>0$.

Proof. Assume $u_{n} \rightarrow u_{0}$ weakly in $V$ and

$$
\limsup _{n \rightarrow \infty}\left(J\left(u_{n}\right)+\lambda G_{2}\left(u_{n}\right), u_{n}-u_{0}\right)_{V} \leq 0 .
$$

Then we have

$$
\begin{aligned}
0 \geq & \limsup _{n \rightarrow \infty}\left(\left(J\left(u_{n}\right)-J\left(u_{0}\right), u_{n}-u_{0}\right)_{V}+\lambda\left(G_{2}\left(u_{n}\right)-G_{2}\left(u_{0}\right), u_{n}-u_{0}\right)_{V}\right) \\
= & \limsup _{n \rightarrow \infty}\left\{\int\left(\left|\nabla u_{n}\right|^{p-2} \nabla u_{n}-\left|\nabla u_{0}\right|^{p-2} \nabla u_{0}\right)\left(\nabla u_{n}-\nabla u_{0}\right)\right. \\
& \left.+\lambda \int g_{2}(x)\left(\left|u_{n}\right|^{p-2} u_{n}-\left|u_{0}\right|^{p-2} u_{0}\right)\left(u_{n}-u_{0}\right)\right\} .
\end{aligned}
$$

We then derive from (3.1) and (2.12) that

$$
\int\left|\nabla u_{n}\right|^{p} \rightarrow \int\left|\nabla u_{0}\right|^{p}, \quad \int g_{2}(x)\left|u_{n}\right|^{p} \rightarrow \int g_{2}(x)\left|u_{0}\right|^{p} .
$$

This together with weak convergence of $u_{n} \rightarrow u_{0}$ in $V$ implies that $\nabla u_{n} \rightarrow \nabla u_{0}$ in $L^{p}\left(R^{N}\right)$. This is also to say $u_{n} \rightarrow u_{0}$ in $V$ if $p \geq N$. For the case $1<p<N$, it follows from Hardy's inequality that

$$
\int \frac{\left|u_{n}-u_{0}\right|^{p}}{(1+|x|)^{p}} \leq\left(\frac{p}{N-p}\right)^{p} \int\left|\nabla\left(u_{n}-u_{0}\right)\right|^{p} \rightarrow 0 .
$$

We again conclude that $u_{n} \rightarrow u_{0}$ in $V$. This ends the proof.

It follows from Lemma 3.3 that

$$
\operatorname{Deg}[J-\lambda G-F(\lambda, \cdot) ; D, 0]
$$

(where $D$ is a bounded open set in $V$ such that $A_{\lambda}(u) \neq 0$ for any $u \in \partial D$ ) is well defined for any $\lambda>0$.

Remark 3.3. If $g \in L^{\infty}\left(R^{N}\right) \cap L^{N / p}\left(R^{N}\right)(1<p<N)$ then $G$ is compact (cf. Lemma 2.2 and the proof of compactness of $G_{1}$ ) and the degree (3.2) can be defined also for $\lambda \leq 0$. 


\section{Bifurcation From $\lambda_{1}$}

Definition 4.1. Let $E=R \times V$ be equipped with the norm

$$
\|(\lambda, u)\|_{E}=\left(|\lambda|^{2}+\|u\|_{V}^{2}\right)^{1 / 2}, \quad(\lambda, u) \in E .
$$

We say that

$$
C=\{(\lambda, u) \in E:(\lambda, u) \text { solves }(2.1), u \neq 0\}
$$

is a continuum of nontrivial solutions of (2.1) if it is a connected set in $E$ with respect to the topology induced by the norm (4.1). We say $\lambda_{0} \in R$ is a bifurcation point of (2.1) (in the sense of Rabinowitz) if there is a continuum of nontrivial solutions $C$ of (2.1) such that $\left(\lambda_{0}, 0\right) \in \bar{C}$ and $C$ is either unbounded in $E$ or there is an eigenvalue $\hat{\lambda} \neq \lambda_{0}$ such that $(\hat{\lambda}, 0) \in \bar{C}$.

Theorem 4.1. Let $1<p<N$. Assume (f1)-(f3) and $g_{1} \in L^{\infty}\left(R^{N}\right) \cap L^{N / p}\left(R^{N}\right)$, $g_{1} \not \equiv 0$. Then the principal eigenvalue $\lambda_{1}^{+}>0$ of the eigenvalue problem

$$
-\Delta_{p} u=\lambda g|u|^{p-2} u, \text { in } R^{N}, \int g|u|^{p}>0
$$

is a bifurcation point of (2.1).

Proof. The proof consists of three steps, the last one being only a variation of the proof of Rabinowitz's theorem [R, Theorem 1.3].

Step 1. First consider the operator $\tilde{A}_{\lambda}(u)=J(u)-\lambda G(u)$. It follows from the variational characteristic of $\lambda_{1}^{+}$that for $\lambda \in\left(0, \lambda_{1}^{+}\right)$and any $u \in V$ with $\|u\|_{V} \neq 0$, we have

$$
\left(\tilde{A}_{\lambda}(u), u\right)_{V}>0 .
$$

Then the degree

$$
\operatorname{Deg}\left[\tilde{A}_{\lambda} ; B_{r}(0), 0\right]
$$

is well defined for any $\lambda \in\left(0, \lambda_{1}\right)$ and any ball $B_{r}(0) \subset V$. Applying Lemma 3.2 we get

$$
\operatorname{Deg}\left[\tilde{A}_{\lambda} ; B_{r}(0), 0\right]=1, \lambda \in\left(0, \lambda_{1}^{+}\right) .
$$

According to Lemma 2.3 there exists a $\delta>0$ such that the interval $\left(\lambda_{1}^{+}, \lambda_{1}^{+}+\delta\right)$ does not contain any eigenvalue of the problem (2.1). Hence the degree (4.2) is well defined also for $\lambda \in\left(\lambda_{1}^{+}, \lambda_{1}^{+}+\delta\right)$. To evaluate Ind $\left(\tilde{A}_{\lambda}, 0\right)$ for $\lambda \in\left(\lambda_{1}^{+}, \lambda_{1}^{+}+\delta\right)$, we use similar procedure as in [D1, D2].

Fix a $K>0$ and define a function $\psi: R \rightarrow R$ by

$$
\psi(t)= \begin{cases}0, & \text { for } t \leq K, \\ \frac{2 \delta}{\lambda_{1}^{+}}(t-2 K), & \text { for } t \geq 3 K,\end{cases}
$$

and $\psi(t)$ is positive and strictly convex in $(K, 3 K)$. We define the functional

$$
\Psi_{\lambda}(u)=\frac{1}{p}(J(u), u)_{V}-\frac{\lambda}{p}(G(u), u)_{V}+\psi\left(\frac{1}{p}(J(u), u)_{V}\right) .
$$

Then $\Psi_{\lambda}$ is continuously Fréchet differentiable and its critical point $u_{0} \in V$ corresponds to the solution of the equation

$$
J\left(u_{0}\right)-\frac{\lambda}{1+\psi^{\prime}\left(\frac{1}{p}\left(J\left(u_{0}\right), u_{0}\right)_{V}\right)} G\left(u_{0}\right)=0 .
$$


However, since $\lambda \in\left(\lambda_{1}^{+}, \lambda_{1}^{+}+\delta\right)$, the only nontrivial critical points of $\Psi_{\lambda}^{\prime}$ occur if

$$
\psi^{\prime}\left(\frac{1}{p}\left(J\left(u_{0}\right), u_{0}\right)_{V}\right)=\frac{\lambda}{\lambda_{1}^{+}}-1,
$$

and hence we must have

$$
\frac{1}{p}\left(J\left(u_{0}\right), u_{0}\right)_{V} \in(K, 3 K) .
$$

In this case either $u_{0}=-u_{1}^{+}$or $u_{0}=u_{1}^{+}$, where $u_{1}^{+}>0$ is the principal eigenfunction. So, for $\lambda \in\left(\lambda_{1}^{+}, \lambda_{1}^{+}+\delta\right)$ the derivative $\Psi_{\lambda}^{\prime}$ has precisely three isolated critical points $-u_{1}^{+}, 0, u_{1}^{+}$. The functional $\Psi_{\lambda}$ is weakly lower semicontinuous. In fact, assume $u_{n} \rightarrow u_{0}$ weakly in $V$. Then

$$
\left(G_{1}\left(u_{n}\right), u_{n}\right)_{V} \rightarrow\left(G_{1}\left(u_{0}\right), u_{0}\right)_{V}
$$

due to the compactness of $G_{1}$ (Lemma 2.2), and

$$
\begin{gathered}
\liminf _{n \rightarrow \infty}\left(\frac{1}{p}\left(J\left(u_{n}\right), u_{n}\right)_{V}+\frac{\lambda}{p}\left(G_{2}\left(u_{n}\right), u_{n}\right)_{V}+\psi\left(\frac{1}{p}\left(J\left(u_{n}\right), u_{n}\right)_{V}\right)\right) \\
\geq \frac{1}{p}\left(J\left(u_{0}\right), u_{0}\right)_{V}+\frac{\lambda}{p}\left(G_{2}\left(u_{0}\right), u_{0}\right)_{V}+\psi\left(\frac{1}{p}\left(J\left(u_{0}\right), u_{0}\right)_{V}\right),
\end{gathered}
$$

by the facts that $\liminf \left\|u_{n}\right\|_{V} \geq\left\|u_{0}\right\|_{V}, \liminf \left\|\nabla u_{n}\right\|_{L^{p}\left(R^{N}\right)} \geq\left\|\nabla u_{0}\right\|_{L^{p}\left(R^{N}\right)}$ and that $\psi$ is nondecreasing.

The relations (4.4) and (4.5) then imply

$$
\liminf _{n \rightarrow \infty} \Psi_{\lambda}\left(u_{n}\right) \geq \Psi_{\lambda}\left(u_{0}\right) .
$$

Observe that $\Psi$ is coercive, i.e.,

$$
\lim _{\|u\|_{V} \rightarrow \infty} \Psi_{\lambda}(u)=\infty .
$$

Indeed, observe first that for $\|u\|_{V} \rightarrow \infty$ two cases can occur: (i) $(J(u), u)_{V}$ is bounded and hence $\left(G_{1}(u), u\right)_{V}$ is bounded too, by the Sobolev embedding, but $\left(G_{2}(u), u\right)_{V} \rightarrow \infty$; (ii) $(J(u), u)_{V} \rightarrow \infty$. In the former case clearly $\Psi_{\lambda}(u) \rightarrow \infty$ for $\|u\|_{V} \rightarrow \infty$. In the latter case we can estimate $\Psi_{\lambda}(u)$ as follows:

$$
\begin{aligned}
\Psi_{\lambda}(u) & =\frac{1}{p}(J(u), u)_{V}-\frac{\lambda_{1}^{+}}{p}(G(u), u)_{V}+\frac{\lambda_{1}^{+}-\lambda}{p}(G(u), u)_{V}+\psi\left(\frac{1}{p}(J(u), u)_{V}\right) \\
& \geq \frac{\lambda_{1}^{+}-\lambda}{p}(G(u), u)_{V}+\psi\left(\frac{1}{p}(J(u), u)_{V}\right) \\
& \geq \frac{\lambda_{1}^{+}-\lambda}{p \lambda_{1}^{+}}(J(u), u)_{V}+\psi\left(\frac{1}{p}(J(u), u)_{V}\right) \\
& \geq-\frac{\delta}{p \lambda_{1}^{+}}(J(u), u)_{V}+\frac{2 \delta}{\lambda_{1}^{+}}\left[\frac{1}{p}(J(u), u)_{V}-2 K\right] \rightarrow \infty,
\end{aligned}
$$

for $\|u\|_{V} \rightarrow \infty$. Here we used the variational characterization for $\lambda_{1}^{+}$and the definition of $\psi$. Since $\Psi_{\lambda}$ is even, there are precisely two points at which the minimum of $\Psi_{\lambda}$ is achieved: $-u_{1}^{+}, u_{1}^{+}$. The point 0 is obviously an isolated critical point of "the saddle type." By virtue of Lemma 3.1, we have

$$
\text { Ind }\left(\Psi_{\lambda}^{\prime},-u_{1}^{+}\right)=\operatorname{Ind}\left(\Psi_{\lambda}^{\prime}, u_{1}^{+}\right)=1 \text {. }
$$

Simultaneously, we have that $\left(\Psi_{\lambda}^{\prime}(u), u\right)_{V}>0$ for any $u \in V,\|u\|_{V}=\kappa$ with $\kappa>0$ large enough. Here we have to consider again separately two cases as in the proof 
of coerciveness of $\Psi_{\lambda}$. In the case (i) clearly again $\left(\Psi_{\lambda}^{\prime}(u), u\right)_{V} \rightarrow \infty$. In the case (ii) we use the following estimate:

$$
\begin{aligned}
\left(\Psi_{\lambda}^{\prime}(u), u\right)_{V}= & (J(u), u)_{V}-\lambda(G(u), u)_{V}+\psi^{\prime}\left(\frac{1}{p}(J(u), u)_{V}\right)(J(u), u)_{V} \\
= & (J(u), u)_{V}-\lambda_{1}^{+}(G(u), u)_{V} \\
& +\psi^{\prime}\left(\frac{1}{p}(J(u), u)_{V}\right)\left[(J(u), u)_{V}-\frac{\lambda-\lambda_{1}^{+}}{\psi^{\prime}\left(\frac{1}{p}(J(u), u)_{V}\right)}(G(u), u)_{V}\right] \\
\geq & \frac{2 \delta}{\lambda_{1}^{+}}\left[(J(u), u)_{V}-2 K\right] \cdot\left[(J(u), u)_{V}-\frac{\lambda_{1}^{+}}{2}(G(u), u)_{V}\right] \rightarrow \infty
\end{aligned}
$$

for $\|u\|_{V} \rightarrow \infty$. We have used again the variational characterization of $\lambda_{1}^{+}$and the definition of $\psi$. Lemma 3.2 then implies that

$$
\operatorname{Deg}\left[\Psi_{\lambda}^{\prime} ; B_{\kappa}(0), 0\right]=1 .
$$

We choose $\kappa$ so large that $\pm u_{1}^{+} \in B_{\kappa}(0)$. Now, by the additivity of the degree (see $[\mathrm{SK}]$ ), and (4.6) and (4.7), we have

$$
\text { Ind }\left(\Psi_{\lambda}^{\prime}, 0\right)=-1 \text {. }
$$

We further have, by the definition of $\psi$,

$$
\operatorname{Deg}\left[\tilde{A}_{\lambda} ; B_{r}(0), 0\right]=\operatorname{Ind}\left(\Psi_{\lambda}^{\prime}, 0\right)
$$

for $r>0$ small enough. We then conclude from (4.3), (4.8) and (4.9) that

$$
\begin{aligned}
& \text { Ind }\left(\tilde{A}_{\lambda}, 0\right)=1, \lambda \in\left(0, \lambda_{1}^{+}\right), \\
& \text {Ind }\left(\tilde{A}_{\lambda}, 0\right)=-1, \lambda \in\left(\lambda_{1}^{+}, \lambda_{1}^{+}+\delta\right) .
\end{aligned}
$$

Step 2. It follows from (2.2) and the homotopy invariance of the degree that for $r>0$ small enough,

$$
\operatorname{Deg}\left[A_{\lambda} ; B_{r}(0), 0\right]=\operatorname{Deg}\left[\tilde{A}_{\lambda} ; B_{r}(0), 0\right]
$$

for $\lambda \in\left(0, \lambda_{1}^{+}+\delta\right) \backslash\left\{\lambda_{1}^{+}\right\}$. We have, from (4.10),

$$
\begin{aligned}
& \text { Ind }\left(A_{\lambda}, 0\right)=1, \lambda \in\left(0, \lambda_{1}^{+}\right), \\
& \text {Ind }\left(A_{\lambda}, 0\right)=-1, \lambda \in\left(\lambda_{1}^{+}, \lambda_{1}^{+}+\delta\right) .
\end{aligned}
$$

Step 3. Following the proof of Theorem 1.3 in $[\mathrm{R}]$ we can prove the conclusion of this theorem.

This completes the proof.

Theorem 4.2. Assume (f1)-(f3), $g \in L^{\infty}\left(R^{N}\right) \cap L^{N / p}\left(R^{N}\right)$ and $g^{ \pm} \not \equiv 0$. Then the conclusion of Theorem 4.1 remains valid. Moreover the principal eigenvalue $\lambda_{1}^{-}<0$ of the eigenvalue problem

$$
-\Delta_{p} u=\lambda g|u|^{p-2} u, \text { in } R^{N}, \quad \int g|u|^{p}<0
$$

is a bifurcation point of (2.1).

Proof. Under the assumptions of this theorem, the operator $G_{2}$ is also compact. So, substituting $-g$ for $g$ in (2.1), Theorem 4.1 is applicable. This concludes the proof. 
Similarly, for the case $p \geq N$, modifying the assumptions in the spirit of Remark 2.1, we have the following analogy of Theorem 4.1.

Theorem 4.3. Let $p \geq N$. Assume (f1)-(f3), $g_{1} \in L^{\infty}\left(R^{N}\right) \cap L^{N_{0} / p}\left(R^{N}\right), g_{1} \not \equiv 0$ for some $N_{0}>p$, and $g_{2}(x) \geq \varepsilon>0$ in $R^{N}$. Then the principal eigenvalue $\lambda_{1}^{+}>0$ of the eigenvalue problem

$$
-\Delta_{p} u=\lambda g|u|^{p-2} u, \text { in } R^{N}, \int g|u|^{p}>0
$$

is a bifurcation point of (2.1).

Remark 4.1. We note that, for the $p$-Laplacian with $p \neq 2$, bifurcation from the principal eigenvalue on a bounded domain has been studied in [DM, D1, D2] for positive weight function, and in $[\mathrm{BH}]$ for indefinite weight function, by calculating the corresponding topological degrees.

Remark 4.2. For $p=2$, $[\mathrm{ER}]$ and $[\mathrm{RE}]$ considered the following bifurcation problem in $R^{N}$ :

$$
\Delta u+\lambda f(x)(u+h(u))=0,
$$

where $f(x)>0$ satisfying certain growth condition (comparable to that of ours on $\left.g_{1}(x)\right)$, and $h(u)$ satisfies

$$
\lim _{t \rightarrow 0^{+}} \frac{h(t)}{t}=a>-1 .
$$

Observe that the effect of $h(u)$ only shifts the principal eigenvalue from $\lambda_{1}^{+}$to $\frac{\lambda_{1}}{a+1}$, so our result essentially contains Theorem 6 of [RE], and we allow $g(x)$ to change sign. We also note that since the utilization of Green's function is fundamental in their calculation of the topological degrees, their method cannot be extended to the case $p \neq 2$.

Remark 4.3. Assuming

$$
\lim _{u \rightarrow \infty} \frac{f(\lambda, x, u)}{w(x)|u|^{p-2} u}=0,
$$

which corresponds to assuming $h(t)$ is bounded in [RE], we can discuss bifurcation from the infinity. Indeed, let $v=u \cdot\|u\|_{V}^{-p /(p-1)}$. Then $u=v \cdot\|v\|_{V}^{-p}$. Multiplying equation (2.1) by $\|u\|_{V}^{-p}$ we get

$$
-\Delta_{p} v=\lambda g(x)|v|^{p-2} v+\|v\|_{V}^{p(p-1)} f\left(\lambda, x,\|v\|_{V}^{-p} v\right) .
$$

Observe that

$$
\frac{\|v\|_{V}^{p(p-1)} f\left(\lambda, x,\|v\|_{V}^{-p} v\right)}{w(x)|v|^{p-2} v}=\frac{f(\lambda, x, u)}{w(x)|u|^{p-2} u} \rightarrow 0
$$

as $v \rightarrow 0$. Thus (f3) is satisfied for (4.11). Now define a new operator $\hat{A}_{\lambda}$ as

$$
\hat{A}_{\lambda}(v)=\frac{A_{\lambda}(u)}{\|u\|_{V}^{p}}=J(v)-\lambda G(v)-\|v\|_{V}^{p(p-1)} F\left(\lambda,\|v\|_{V}^{-p} v\right) .
$$

We see that the calculations in the proof of Theorem 4.1 remain valid for $\hat{A}_{\lambda}$; hence $\left(\lambda_{1}^{+}, 0\right)$ is a bifurcation point for the problem associated with $\hat{A}_{\lambda}$. Consequently $\left(\lambda_{1}^{+}, \infty\right)$ is a bifurcation point of (2.1). This is compatible with Theorem 9 of [RE].

We want to further strengthen our bifurcation result. Instead of (f2) we assume 
(f2 $)^{\prime}$ There is a nonnegative $\tilde{\rho}(x) \in L^{\infty}\left(R^{N}\right) \cap L^{\gamma_{1}}\left(R^{N}\right)$ such that

$$
|f(\lambda, x, u)| \leq c(\lambda) \tilde{\rho}(x)|u|^{\gamma}
$$

for all $\lambda, u \in R$ and a.e. $x \in R^{N}$.

Then we have the following result.

Theorem 4.4. Let $1<p<N$, and assume (f1), (f2)', (f3) hold, $g_{1} \in L^{\infty}\left(R^{N}\right) \cap$ $L^{N / p}\left(R^{N}\right)$ and $g_{1} \not \equiv 0$. Then for any solution $u$ of $(2.1)$ with $\lambda \geq 0, u \in L^{Q}\left(R^{N}\right)$ for any $p^{*} \leq Q \leq \infty$ and $u$ decays uniformly as $|x| \rightarrow \infty$. Moreover, $u \in$ $C^{1, \alpha}\left(B_{K}(0)\right)$ for any $K>0$ with some $\alpha=\alpha(K) \in(0,1)$.

Proof. The proof of $u^{ \pm} \in L^{Q}\left(R^{N}\right), p^{*} \leq Q \leq \infty$, and the decay of $u^{ \pm}$as $|x| \rightarrow \infty$ can be performed in the similar way as in the steps 2,3 of the proof of Theorem 3.1 in [D3], by separating $u^{+}$and $u^{-}$and using the bootstrap argument and Serrin's estimates (cf. [SE]). The regularity result follows then from [TO]. Note that here $u^{ \pm}$denotes the positive and negative part of $u$ respectively.

Let $\varphi^{*} \in V^{*}$ be fixed and satisfy

$$
\left(\varphi^{*}, u_{1}^{+}\right)_{V}=1
$$

and for $\tau \in(0,1)$ define the sets

$$
\begin{aligned}
& K_{\tau}^{+}=\left\{(\lambda, u) \in E:\left(\varphi^{*}, u\right)_{V}>\tau\|u\|_{V}\right\}, \\
& K_{\tau}^{-}=\left\{(\lambda, u) \in E:\left(\varphi^{*}, u\right)_{V}<-\tau\|u\|_{V}\right\} .
\end{aligned}
$$

Note that $K_{\tau}^{ \pm}$are cones containing $\pm u_{1}^{+}$respectively, and $\tau$ is a measure of "openness" of these cones. Furthermore, let $B_{\eta}\left(\lambda_{1}^{+}, 0\right)$ denote the ball in $E$ centered at the point $\left(\lambda_{1}^{+}, 0\right)$ with radius $\eta$.

Theorem 4.5. Let the assumptions of Theorem 4.4 be fulfilled. Moreover, suppose that there exists $\delta>0$ such that

$$
J(u)=\lambda_{1}^{+} G(u)+F\left(\lambda_{1}^{+}, u\right)
$$

has no nonzero solution $u \in V, 0<\|u\|_{V}<\delta$. Then there are maximal connected subsets $C^{+}, C^{-}$of $C\left(C\right.$ is the set from Theorem 4.1) containing $\left(\lambda_{1}^{+}, 0\right) \in E$ in their closures,

$$
C^{ \pm} \cap B_{\eta}\left(\lambda_{1}^{+}, 0\right) \subset K_{\tau}^{ \pm}, \quad \eta=\eta(\tau) \rightarrow 0 \text { as } \tau \rightarrow 1,
$$

and such that both $C^{ \pm}$are unbounded in $E$. Moreover, $\lambda>\lambda_{1}^{+}$for any $(\lambda, u) \in C^{ \pm}$, $u>0$ in $R^{N}$ if $(\lambda, u) \in C^{+}$, and $u<0$ in $R^{N}$ if $(\lambda, u) \in C^{-}$.

Proof. Following step by step of the proof of Theorem 2 in [DA], we get the existence of the maximal connected subsets $C^{ \pm} \subset C$ satisfying (4.13) and being either unbounded in $E$ or containing in their closures a common point different from $\left(\lambda_{1}^{+}, 0\right) \in E$ (cf. [D2]). We will show that the latter case cannot occur.

Note that $\lambda>\lambda_{1}^{+}$for any $(\lambda, u) \in C^{ \pm}$due to the fact that (4.12) has no nonzero solution for $\lambda=\lambda_{1}^{+}$. Let $\left(\lambda_{n}, u_{n}\right) \in C^{+}$be a sequence such that $\lambda_{n} \rightarrow \lambda_{1}^{+}$. Then $\left\|u_{n}\right\|_{V} \rightarrow 0$. Denoting $\tilde{u}_{n}=u_{n} \cdot\left\|u_{n}\right\|_{V}^{-1}$ we can assume due to (f2)' and (f3) that $\tilde{u}_{n} \rightarrow u_{1}^{+}$weakly in $V$. Similarly as in the proof (ii) of Lemma 2.3 we 
derive that $\tilde{u}_{n} \rightarrow u_{1}^{+}$strongly in $V$. We claim that for $n$ large, $u_{n}>0$. Denote $\Omega_{n}^{-}=\left\{x \in R^{N} ; \tilde{u}_{n}(x)<0\right\}$. Then we have

$$
\int\left|\nabla u_{n}^{-}\right|^{p}+\lambda \int g_{2}(x)\left|u_{n}^{-}\right|^{p}=\lambda \int g_{1}(x)\left|u_{n}^{-}\right|^{p}+\int f\left(\lambda, x, u_{n}^{-}\right) u_{n}^{-} .
$$

By Hardy's, Hölder's, Sobolev's inequalities, the assumptions on $g$ and (f2)', we get

$$
c_{15}\left\|u_{n}^{-}\right\|_{V}^{p} \leq c_{16}\left(\int_{\Omega_{n}^{-}}\left(g_{1}(x)\right)^{N / p}\right)^{p / N}\left\|u_{n}^{-}\right\|_{V}^{p}+c_{17}\left(\int_{\Omega_{n}^{-}}(\tilde{\rho}(x))^{\gamma_{1}}\right)^{1 / \gamma_{1}}\left\|u_{n}^{-}\right\|_{V}^{\gamma+1},
$$

i.e.

$$
c_{15} \leq c_{16}\left(\int_{\Omega_{n}^{-}}\left(g_{1}(x)\right)^{N / p}\right)^{p / N}+c_{17}\left(\int_{\Omega_{n}^{-}}(\tilde{\rho}(x))^{\gamma_{1}}\right)^{1 / \gamma_{1}}\left\|u_{n}^{-}\right\|_{V}^{\gamma+1-p} .
$$

Since $\left\|u_{n}\right\|_{V} \rightarrow 0, g_{1} \in L^{N / p}\left(R^{N}\right), \tilde{\rho} \in L^{\gamma_{1}}\left(R^{N}\right)$ and $c_{15}, c_{16}, c_{17}$ do not depend on $u_{n}$, we derive from here that for $K_{0}>0$ large enough,

$$
\text { meas }\left(\Omega_{n}^{-} \cap B_{K}(0)\right) \geq c_{18}
$$

for any $K>K_{0}$, where $c_{18}>0$ depends on neither $\lambda_{n}$ nor $u_{n}$. Now, using the same argument as in the proof (ii) of Lemma 2.3 based on the Egorov Theorem we deduce that $\tilde{u}_{n}$ (and hence $u_{n}$ ) is nonnegative in $R^{N}$ for $n$ large enough. It then follows that $u \geq 0$ for any $(\lambda, u) \in C^{+} \cap B_{\eta}\left(\lambda_{1}^{+}, 0\right)$ with $\eta>0$ small enough.

Assume now that there exists $(\hat{\lambda}, \hat{u}) \in C^{+}$such that $\hat{u}(x) \leq 0$ at some point $x \in R^{N}$. Since $C^{+}$is connected and by the $C_{\mathrm{loc}}^{1, \alpha}$-regularity of solutions of (2.1), there exists $\left(\lambda^{*}, u^{*}\right) \in C$ such that $u^{*} \not \equiv 0, u^{*} \geq 0$, and $u^{*}\left(x_{0}\right)=0$ for some $x_{0} \in R^{N}$. This violates the Harnack inequality (see Theorem 1.2 in [TR]). Hence $u>0$ for any $(\lambda, u) \in C^{+}$and similarly $u<0, \lambda>\lambda_{1}^{+}$for all $(\lambda, u) \in C^{-}$. This excludes the second alternative of Theorem 2 in [DA] and thus $C^{ \pm}$must be unbounded in $E$. This ends the proof.

Remark 4.4. For the case $p>N$, any solution $u$ of $(2.1)$ is in $L^{\infty}\left(R^{N}\right)$ by Sobolev's embedding theorem, while for $p=N$, solution of (2.1) belongs to $L^{r}\left(R^{N}\right)$ for $p \leq r<\infty$. Observe that in either case changing the assumptions in the spirit of Remark 2.1, the arguments in the proofs of Theorems 4.4 and 4.5 are still valid, so Theorems 4.4 and 4.5 remain true for $p \geq N$.

Remark 4.5. For $1<p<N$, if (f1), (f2 $)^{\prime}$, (f3) hold, $g \in L^{\infty}\left(R^{N}\right) \cap L^{N / p}\left(R^{N}\right)$ and $g^{ \pm} \not \equiv 0$, then the same conclusions of Theorems 4.4 and 4.5 also hold for $\lambda_{1}^{-}$.

Remark 4.6. One possible form of $f(\lambda, x, u)$ satisfying the hypothesis of Theorem 4.5 is the following:

$$
f(\lambda, x, u)=-c(\lambda) \tilde{\rho}(x)|u|^{\gamma-1} u,
$$

where $c\left(\lambda_{1}^{+}\right)>0$ and $\tilde{\rho}(x)>0$ for a.e. $x \in R^{N}$. Indeed, due to the variational characterization of $\lambda_{1}^{+}>0$, we have

$$
\int|\nabla u|^{p}-\lambda \int g(x)|u|^{p} \geq 0
$$

and

$$
\int f(\lambda, x, u) u=-c(\lambda) \int \tilde{\rho}(x)|u|^{\gamma+1}<0
$$

for any $\lambda \leq \lambda_{1}^{+}, \lambda$ close to $\lambda_{1}^{+}$and any $u \in V$ with $u \neq 0$. Note that in this case we have $(\lambda, u) \in C^{+}$if and only if $(\lambda,-u) \in C^{-}$. 
Remark 4.7. Suppose that $c\left(\lambda_{0}\right)=0$ (in (f2)') for some $\lambda_{0}>\lambda_{1}^{+}$; then $C^{+}$(and similarly for $C^{-}$) from Theorem 4.5 "blows up" in $\|u\|_{V}$. In fact, since

$$
-\Delta_{p} u=\lambda_{0} g(x)|u|^{p-2} u
$$

cannot have a positive solution in $R^{N}$, the parameter $\lambda$ cannot cross the value $\lambda_{0}$ and hence $\|u\|_{V}$ is unbounded along $C^{+}$.

\section{REFERENCES}

[AD] R.A. Adams, Sobolev Spaces, Academic Press, 1975. MR 56:9247

[AH] W. Allegretto and Y.X. Huang, Eigenvalues of the indefinite weight p-Laplacian in weighted $R^{N}$ spaces, Funkc. Ekvac. 38 (1995), 233-242.

[A] A. Anane, Simplicité et isolation de la première valeur propre du p-laplacien avec poids, C.R. Acad. Sci. Paris 305 I (1987), 725-728. MR 89e:35124

[BH] P.A. Binding and Y.X. Huang, Bifurcation from eigencurves of the p-Laplacian, Diff. Int. Equa. 8 (1995), 415-428. MR 95j:35165

[BP] F.E. Browder and W.F. Petryshyn, Approximation methods and the generalized topological degree for nonlinear mappings in Banach spces, J. Funct. Anal. 3 (1969), 217-245. MR 39:6126

[DA] E.N. Dancer, On the structure of the solutions of nonlinear eigenvalue problems, Indiana Univ. Math. J. 23 (1974), 1069-1076. MR 50:1065

[DM] M.A. Del Pino and R. Manásevich, Global bifurcation from the eigenvalues of the $p$ Laplacian, J, Diff. Equa. 92 (1991), 226-251. MR 92g:35028

[D1] P. Drábek, On the global bifurcation for a class of degenerate equations, Ann. Mat. Pura Appl. 159 (1991), 1-16. MR 93d:47112

[D2] P. Drábek, Solvability and Bifurcations of Nonlinear Equations, Pitman Research Notes in Math. 264, Longman, Harlow, 1992. MR 94e:47084

[D3] P. Drábek, Nonlinear eigenvalue problem for the $p$-Laplacian in $R^{N}$, Math. Nach. 173 (1995), 131-139. MR 96b:35064

[ER] A. Edelson and A. Rumbos, Linear and semilinear eigenvalue problems in $R^{N}$, Comm. Part. Diff. Equa. 18 (1993), 215-240. MR 94b:35101

[FK] S. Fučik and A. Kufner, Nonlinear Differential Equations, Elsevier, Holland, 1980. MR 81e:35001

[HS] E. Hewitt and K. Stromberg, Real and Abstract Analysis, Springer-Verlag, New York, 1975. MR 51:3363

[KA1] Y. Kabeya, Existence theorems for quasilinear elliptic problems in $R^{N}$, Funkc. Ekvac. 35 (1992), 603-616. MR 94b:35103

[KA2] Y. Kabeya, On some quasilinear elliptic problems involving critical Sobolev exponents, Funkc. Ekvac. 36 (1993), 385-404. MR 94k:35106

[KU] I.A. Kuzin, On multiple solvability of some elliptic problems in $R^{N}$, Soviet Math. Dokl. 44 (1992), 700-704.

[LY] G. Li and S. Yan, Eigenvalue problem for quasilinear elliptic equations in $R^{N}$, Comm. Part. Diff. Equa. 14 (1989), 1291-1314. MR 91a:35072

[LQ] P. Lindqvist, On the equation $\operatorname{div}\left(|\nabla u|^{p-2} \nabla u\right)+\lambda|u|^{p-2} u=0$, Proc. Amer. Math. Soc. 109 (1990), 157-164. MR 90h:35088

[R] P. Rabinowitz, Some global results for nonlinear eigenvalue problems, J. Funct. Anal. 7 (1971), 487-513. MR 46:745

[RE] A. Rumbos and A. Edelson, Bifurcation properites of semilinear elliptic equations in $R^{N}$, Diff. Int. Equa. 7 (1994), 399-410. MR 94m:35028

[SC] I. Schindler, Quasilinear elliptic boundary-value problems on unbounded cylinders and a related mountain-pass lemma, Arch. Rat. Mech. Anal. 120 (1992), 363-374. MR 93k:35093

[SE] J. Serrin, Local behavior of solutions of quasilinear equations, Acta Math. 111 (1964), 247302. MR 30:3327

[SK] I.V. Skrypnik, Nonlinear Elliptic Equations of Higher Order (in Russian), Naukovaja Dumka, Kyjev, 1973. MR 55:8549 Nonlinear Elliptic Boundary Value Problems (in English), Teubner Texte fur Math. 91, Teubner-Verlag, Leipzig, 1986. MR 89g:35040

[TO] P. Tolksdorf, Regularity for a more general class of quasilinear elliptic equations, J. Diff. Equa. 51 (1984), 126-150. MR 85g:35047 
[TR] N.S. Trudinger, On Harnack type inequalities and their application to quasilinear elliptic equations, Comm. Pure Appl. Math. 20 (1967), 721-747. MR 37:1788

[Y] L.S. Yu, Nonlinear p-Laplacian problems on unbounded domains, Proc. Amer. Math. Soc. 115 (1992), 1037-1045. MR 93e:35027

Department of Mathematics, University of West Bohemia, P.O. Box 314, 30614 Pilsen, Czech Republic

Department of Mathematical Sciences, University of Memphis, Memphis, Tennessee 38152

E-mail address: huangy@mathsci.msci.memphis.edu 